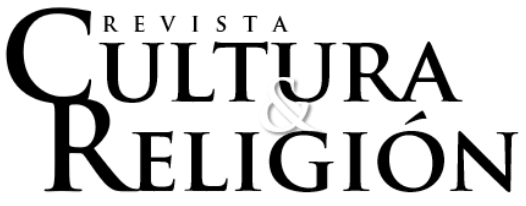

Vol. XV, № 2 (2021) pp. 244-272

Recibido: 15 de octubre de 2020

Aceptado: 30 de julio de 2021

\title{
IDENTIDAD, CONVIVENCIA Y ESPIRITUALIDAD EN UNA COMUNIDAD ECOLÓGICA HARE KRISHNA*
}

\author{
Identity, coexistence and spirituality in a Hare Krishna ecological \\ community
}

\author{
Rodolfo E. Mardones ${ }^{* *}$ \\ Universidad Austral de Chile, Chile \\ ORCID: 0000-0003-4027-1027 \\ Paula Díaz Castillo ${ }^{* * *}$ \\ Universidad Austral de Chile, Chile \\ ORCID: 0000-0003-0315-7583
}

\section{Resumen}

El objetivo de este artículo es comprender la construcción de la identidad de los integrantes de una comunidad intencional Hare Krishna. Seleccionamos, como caso de estudio, una comunidad ubicada en el sur de Chile debido a su interés por el cambio personal y espiritual. Desplegamos una aproximación etnográfica que consideró observación participante, conversaciones cotidianas y entrevistas semiestructuradas con devotos Hare Krishna. Entre los principales hallazgos de

\footnotetext{
* Artículo asociado al proyecto: DID S-2018-15 “Quiebres ontológicos en la relación comunidad-naturaleza. ¿Del sujeto económico al sujeto psicológico-espiritual?” (mayo 2018 a diciembre 2020), financiado por la VIDCA, Universidad Austral de Chile.

** Doctor en Ciencias Sociales. Profesor en el Instituto de Estudios Psicológicos, Universidad Austral de Chile. Valdivia, Chile. Correo electrónico: rodolfo.mardones@uach.cl

*** Licenciada en Psicología y Psicóloga, Universidad Austral de Chile. Asistente de investigación proyecto VIDCA UACh, S-2018-15. Correo electrónico: paula.diazcas@gmail.com
} 
la investigación identificamos que el eje articulador de la construcción de la identidad de los devotos es la actitud de servicio, la cual se traduce en un conjunto de prácticas para configurar su visión de sociedad y la relación con los otros. Concluimos que la actitud de servicio sostiene prácticas para adorar a Krishna, y al mismo tiempo busca transformar las relaciones con la humanidad, la biodiversidad y consigo mismos.

Palabras clave: identidad, comunidades intencionales, espiritualidad, ecoaldea, cambio social.

\begin{abstract}
The objective of this article is to understand the construction of the identity of the members of an intentional Hare Krishna community. We selected a community located in southern Chile as our case study due it its interest in personal and spiritual change. We deployed an ethnographic approach that included participant observation, daily conversations and semi-structured interviews with Hare Krishna devotees. The main findings of the research include that the articulating axis of the construction of devotees' identity is the service attitude, which translates into a set of practices for configuring their vision of society and relationship to others. We conclude that the service attitude sustains practices for adoring Krishna and seeks to transform relationships with humanity, biodiversity and themselves.
\end{abstract}

Keywords: Identity, intentional communities, spirituality, eco-village, social change.

\title{
Introducción
}

La identidad es uno de los conceptos de mayor tematización en las ciencias sociales. Muchas son las perspectivas que han esbozado definiciones biologicistas, psicológicas, socioculturales y/o interaccionistas (ÁlvarezMunárriz, 2011; Iñiguez-Rueda, 2001). En este trabajo, entendemos la

Mardones, R. y Díaz, P. (2021), Identidad, convivencia y espiritualidad en una comunidad ecológica Hare Krishna. Revista Cultura y Religión, 15(2), 244-272. 
identidad como un proceso que no se puede interpretar exclusivamente desde la actividad psíquica de los individuos (Rose, 2019), sino que debe considerar las dinámicas sociales caracterizadas por un predominio de la racionalidad económica en todas las dimensiones de la vida de las personas y del planeta (Beriain, 1996; De la Cuadra, 2015). En la actualidad persiste una lógica que intensifica la racionalidad del progreso y el crecimiento económico aparentemente sin límites (Dabat, 2002). A partir de esto, vemos que la humanidad comienza a verse envuelta en una crisis sistémica. Producto de la globalización y la obstinada aspiración progresista, se ha mundializado la crisis financiera, económica, ecológica, alimentaria, social, identitaria y espiritual (Castells, 1999; Estermann, 2012; Nuño, 2017).

Aquí, abordaremos particularmente la construcción de la identidad en el contexto de la fragmentación y fractura de las narrativas universales (Hall, 2011), lo cual ha impulsado a los contemporáneos a buscar nuevas perspectivas y estilos de vida. En consecuencia, la percepción y construcción de la identidad actual se presenta como una estructura dinámica y creativa, en constante cambio y transformación, que se construye y se vive dentro de un medio físico concreto, una contingencia y una época histórica determinada, mediante diferentes discursos y prácticas, a menudo entrecruzadas y antagónicas (Álvarez-Munárriz, 2011; Hall, 2011; Lerner, 2019).

Los pilares de referencia de la sociedad moderna, como la familia, el trabajo y la religión, cambian su estatus de fundamentación segura de la verdad (Ibáñez, 2001) y debilitan su protagonismo como fuentes de sentido para la vida (Taylor, 2006), por lo que el sujeto se constituye más allá de su interioridad psíquica, abriendo sus fronteras de identificación hacia una sociedad globalizada y oscilante, en donde se tiene que identificar con una totalidad abstracta e inestable bajo un contexto de mayor acceso a información, que saturan su subjetividad (Bauman y Tester, 2002; Castells, 1999; Gergen, 2006).

Esta complejidad de lo social estimula que la identidad se constituya en torno a un efecto pluralizador de símbolos lingüísticos difusos, es decir, una construcción nunca acabada, abierta a la temporalidad y a la posicionalidad

Mardones, R. y Díaz, P. (2021), Identidad, convivencia y espiritualidad en una comunidad ecológica Hare Krishna. Revista Cultura y Religión, 15(2), 244-272. 
relacional, produciendo de esta manera una variedad de posibilidades y de nuevas figuras de identificación (Alsina y Bravo, 2006; Schuttenberg, 2007). Esto no significa que se esté cumpliendo la promesa de la modernidad, en relación con la posibilidad de un individuo libre constituido en base de su autoconciencia (Ferreira, 2013), sino que, al contrario, el sujeto experimenta una sensación de angustia al verse expuesto a múltiples interpretaciones de identidad en una sociedad abstracta, oscilante e inestable, donde debe ser responsable de la elección del rumbo de su vida, de sus éxitos y fracasos en este contexto (Gergen, 2006; Thumala, 2003).

En medio de esta crisis sistémica, se ha advertido sobre las consecuencias negativas observables que trajo consigo la ambición progresista, lo que ha hecho surgir un cuestionamiento de las bondades y certezas que prometía la modernidad y una denuncia sobre su perversidad (Beck, Giddens y Lash, 1997; Thumala, 2003). La conciencia de estas consecuencias negativas fortalece el individualismo, ya que el sujeto al verse enfrentado a estas necesita un espacio solitario que le permita cuestionar constantemente su propia transformación y también la del mundo (Álvarez-Munárriz, 2011; Berger y Luckmann, 1997). En efecto, la identidad se forja en una sensación de relatividad, que se sostiene en la escasa posibilidad de establecer una verdad absoluta y objetiva, poniendo en duda hasta la propia identidad construida hasta ese momento (Ibáñez, 2001).

Lo anterior sugiere una dualidad que constriñe al sujeto, pero que al mismo tiempo le entrega la posibilidad de movimiento y búsqueda de nuevas alternativas de vida, que se inician en el sujeto y se extienden a la comunidad y su mundo circundante (Mardones y Zunino, 2019). La identidad y las prácticas cotidianas se entremezclan en una búsqueda utópica altamente individualizada (Sullivan, 2016) y muchas veces de carácter transitorio (Mardones, Aguirre, Aranda y Muñoz, 2019). En esta dinámica, identificamos distintas comunidades más estables en el tiempo que se crean intencionalmente para responder al mundo moderno, sin un relato unitario aparente (Huiliñir-Curío y Zunino, 2017).

Mardones, R. y Díaz, P. (2021), Identidad, convivencia y espiritualidad en una comunidad ecológica Hare Krishna. Revista Cultura y Religión, 15(2), 244-272. 


\section{Revista Cultura y Religión Vol. XV, Nº 2, (julio-diciembre) 2021}

La literatura ha reportado que las comunidades intencionales son un grupo de personas que han elegido vivir juntas cerca de la naturaleza, produciendo y consumiendo a nivel local con un propósito común, en donde se intenta mantener un estilo de vida que refleje sus valores e ideas fundamentales (Kozeny, 1995; Salamanca y Silva, 2015). La principal motivación de estas comunidades es el enfrentamiento a la crisis sistémica actual, y su activismo preponderante está orientado a la transformación social, política y cultural (Silvestri, 2015). Sus convicciones se basan en ideas biocéntricas, que integran perspectivas de transición a la sustentabilidad ecológica, ecología profunda y espiritualidad New Age, o adhesiones religiosas que modulan sus formas de relación (Mardones y Zunino, 2021).

Algunos de estos casos son las comunidades, templos y granjas ecológicas Hare Krishna, las que han logrado consolidarse y expandirse en distintos lugares del mundo y en algunas regiones de Chile, integrando perspectivas ecológicas y espirituales (Bahamondes y Marín, 2015; Farkas, 2018; Mardones y Zunino, 2021).

\section{Espiritualidad Hare Krishna y vida en común}

En la sociedad contemporánea se habla de una religiosidad como sistema abierto de creencias opuesto a la religión institucional (Sánchez, Plascencia y Bernal, 2020), cuya espiritualidad flexible facilita la formación de una identidad en construcción basada en una espiritualidad híbrida y personalizada. Esta se concentra mediante diferentes prácticas espirituales cotidianas, que combinan cosmovisiones provenientes del intercambio cultural entre conocimientos ancestrales y espiritualidades contemporáneas, que se extraen de sus contextos sociohistóricos y culturales para acomodarse a los valores del sujeto (De la Torre, 2014; Farkas, 2018; Muñoz, 2018; Zapata, 2019). En este sentido, la religión personalizada funciona como un proceso dinámico en el que se relativizan las doctrinas, los mensajes, las identidades y los espacios (Nuño, 2017).

Mardones, R. y Díaz, P. (2021), Identidad, convivencia y espiritualidad en una comunidad ecológica Hare Krishna. Revista Cultura y Religión, 15(2), 244-272. 


\section{Revista Cultura y Religión Vol. XV, Nº 2, (julio-diciembre) 2021}

Esta espiritualidad heterogénea se presenta en el movimiento Hare Krishna. En nuestro caso de estudio se vincula con la Asociación Internacional para la Conciencia de Krishna (ISKCON), un movimiento religioso que se inspira en el hinduismo y, aunque con flexibilidades propias de la modernidad occidental, se sostiene en la individualidad del ser humano. Fue fundada por el maestro espiritual Bhaktivedanta Swami Prabhupada en Nueva York, en pleno apogeo del movimiento hippie en la década de 1960, cuya crítica se dirigió a las consecuencias negativas del capitalismo, el consumo, el armamentismo y la falsa felicidad transmitida por los medios de comunicación. Entre los objetivos valóricos del movimiento Hare Krishna se promueve la paz universal, el equilibrio espiritual, el vegetarianismo, la abstención del consumo de drogas y alcohol, además de no mantener relaciones sexuales fuera del matrimonio, ni practicar juegos de azar, como una forma de servir y adorar a su deidad (García, 2018). Este servicio devocional no es visto como una restricción, sino como una aproximación a la felicidad, cuestión que se aproxima al carácter alegre del sacrificio reportado para el caso del cristianismo (Granados, 2020).

En Chile, este movimiento religioso se instaló en los años setenta del siglo pasado. En primera instancia, se fundaron pequeños templos urbanos y, posteriormente, se crearon fincas y comunidades ecológicas para promover una vida de servicio a Krishna (Ávila, 2013; Carnevali, 2009; Quintana, 2011). Su forma de vida parte de una crítica radical de nuestra sociedad y, en consecuencia, considera que cada devoto debe abandonar sus prácticas mundanas para dedicarse al estudio exhaustivo de su libro sagrado (Bhagavad Gita) y trabajar para mantener una cotidianidad de devoción espiritual, lo cual impacta de manera significativa en la definición de sí mismo (Mardones y Zunino, 2021).

Los principales estudios acerca de este movimiento destacan su accionar en la esfera social, cultural, alimentaria y personal. Por ejemplo, se releva la preocupación por la alimentación como una estrategia para responder a las prácticas de nuestra sociedad en esta materia (Alcántara, 2019; Granados, 2019). En un estudio de comunidades Hare Krishna de España, se muestra que

Mardones, R. y Díaz, P. (2021), Identidad, convivencia y espiritualidad en una comunidad ecológica Hare Krishna. Revista Cultura y Religión, 15(2), 244-272. 
existe actualmente una incertidumbre de lo que se consume en nuestra sociedad, debido a la industrialización, cuestión que impulsa a los devotos Hare Krishna a preocuparse de manera activa sobre el origen y los procesos de los alimentos, optando por el vegetarianismo en sus prácticas diarias (Nuño, 2017).

En nuestro país, la atención ha estado puesta en las estrategias de acción social y políticas que el propio movimiento desarrolla para que los devotos se comprometan con la realidad social en la cual se encuentran inmersos. Como un gesto de consecuencia, se incentiva la participación ciudadana con la intención de superar los principios políticos de la democracia liberal (Bahamondes y Marín, 2015).

Por otro lado, se destaca la acción que el movimiento Hare Krishna realiza en la esfera cultural y religiosa; su carácter ecléctico entregado por el relativismo de la postmodernidad facilitan la occidentalización del hinduismo, y permiten la coexistencia de elementos propios de esta época como la racionalidad y el individualismo, junto a la práctica de la meditación o yogas devocionales y posturales, considerados a partir de siglo XX como empíricamente beneficiosos (Luarte, 2017).

Estas preocupaciones no solo se entienden como una actitud devocional dirigida a la sociedad, sino que también responden a una búsqueda de sentido que justifica la motivación de las personas que decidieron convertirse en devotos Hare Krishna (Herrera, 2018). Se advierte que el proceso de conversión de los devotos sigue un modelo gradual que culmina en encontrar un sistema de significados satisfactorios y ajustados a demandas o necesidades individuales, socioemocionales y existenciales (Vallverdú, 1999), así también como una propuesta utópica que contribuye a la búsqueda de su identidad (Squarcini, 2000).

Por ejemplo, las historias de vida de monjes krishnas y los seguidores de Krishna muestran una reconstrucción biográfica drástica, que involucra la adopción de un nuevo patrón de pensamiento y la aceptación de que la conversión solo se logra parcialmente, por lo que se trata de un proceso permanente (Bögre, 2016).

Mardones, R. y Díaz, P. (2021), Identidad, convivencia y espiritualidad en una comunidad ecológica Hare Krishna. Revista Cultura y Religión, 15(2), 244-272. 


\section{Revista Cultura y Religión Vol. XV, Nº 2, (julio-diciembre) 2021}

La literatura nos muestra que los devotos son agentes activos y protagonistas en llevar a cabo esta vida en comunidad, que busca influir en cambios socioculturales y personales relevantes. Sin embargo, su presencia en América Latina ha sido estudiada de manera periférica. En este contexto, nos interesa aproximarnos a la experiencia directa de participantes de una comunidad ecológica espiritual Hare Krishna.

\section{Diseño metodológico}

Nuestra investigación siguió un enfoque cualitativo con un diseño de caso único con el propósito de graficar el fenómeno estudiado (Mardones, Ulloa y Salas, 2018). Nos aproximamos intencionadamente a nuestro caso de estudio (Pérez-Luco, Lagos, Mardones y Sáez, 2017): una comunidad Hare Krishna que se define como un santuario ecológico y espiritual. Se emplaza en la región del Bío-Bio, fue fundada en 2001 y es custodiada por una familia de dos adultos y dos niños. Ahí se realizan actividades espirituales, terapéuticas y de cuidado del medioambiente, lo que promueve la asistencia de decenas de visitantes y voluntarios durante todo el año, los cuales aumentan en los períodos estivales.

Orientados por una perspectiva etnográfica, realizamos visitas a la comunidad, que incluyeron contactos iniciales y luego una estadía durante siete días en febrero de 2019. Con el objetivo de comprender la construcción de identidad de los participantes de esta comunidad, inicialmente seleccionamos de manera intencionada a los integrantes permanentes o guardianes; en este caso, una pareja de dos adultos jóvenes: una mujer de 34 años y un hombre de 27 años, con dos hijos. Una vez que concretamos nuestra estadía en la comunidad y participamos en sus actividades, incluimos la experiencia de dos visitantes y dos voluntarios que se encontraban transitoriamente en la comunidad.

Después de presentar los propósitos de la investigación a los participantes, acordar la confidencialidad y convenir los términos de nuestra

Mardones, R. y Díaz, P. (2021), Identidad, convivencia y espiritualidad en una comunidad ecológica Hare Krishna. Revista Cultura y Religión, 15(2), 244-272. 


\section{Revista Cultura y Religión Vol. XV, Nº 2, (julio-diciembre) 2021}

participación en la comunidad, firmamos un consentimiento informado aprobado por el comité de ética de la Universidad Austral de Chile.

Para aproximarnos a la cotidianidad comunitaria, utilizamos la observación participante; esto es, nos involucramos directamente en las actividades de la comunidad en un contexto cotidiano y natural, para posteriormente tomar notas de campo acerca de ellas (Kawulich, 2005). Para profundizar en las perspectivas de los participantes permanentes, realizamos entrevistas semiestructuradas, las que tomaron la forma de una conversación guiada por preguntas generales (Ulloa y Mardones, 2017). Dichas entrevistas tuvieron dos sesiones de noventa minutos cada una, aproximadamente. Preguntamos por aspectos históricos y administrativos de la comunidad, sobre su forma de organización, las actividades diarias, las formas de participación y su vínculo con el movimiento Hare Krishna; así como aspectos biográficos de los participantes que nos permitieron acercarnos con más detalle a la construcción de su identidad.

Por otra parte, utilizamos conversaciones en lo cotidiano y observación participante (Spink, 2013) para conocer la perspectiva de los visitantes y voluntarios de la comunidad. Entendimos este tipo de conversación como un proceso de diálogo abierto e informal en situaciones naturales de la vida diaria (Erlandson, Harris, Skipper y Allen, 1993); por ejemplo, mientras asistíamos en tareas de aseo, cocina, o en festividades religiosas de la comunidad. Consideramos que los habitantes transitorios o visitantes aportaron información valiosa para comprender las dinámicas de confianza que tienen lugar en la comunidad.

En relación con la organización de los datos, realizamos una descripción que nos permitió contextualizar las palabras de las personas a partir de nuestra observación participante de sus prácticas y conversaciones cotidianas, tanto con los participantes permanentes, como de los visitantes y voluntarios de la comunidad. Por otro lado, realizamos una codificación abierta de las palabras que los participantes permanentes de la comunidad nos compartieron en sus entrevistas, ayudándonos del software de análisis de datos cualitativos Atlas.ti.

Mardones, R. y Díaz, P. (2021), Identidad, convivencia y espiritualidad en una comunidad ecológica Hare Krishna. Revista Cultura y Religión, 15(2), 244-272. 
Posteriormente, realizamos un análisis temático que agrupó los discursos en tres dimensiones que sintetizan prácticas y sentidos para la construcción de identidad de los devotos de esta comunidad: i) directrices espirituales para un cambio sociocultural; ii) convivencia con las personas y la naturaleza, y, iii) espiritualidad Hare Krishna en la definición de sí mismos.

\section{Resultados}

Los participantes de nuestro caso de estudio siguen un conjunto de acciones cotidianas devocionales que mantienen una relación circular con Krishna. Un devoto sirve a Krishna y en este ejercicio de espiritualización busca la iluminación de su conciencia. Para ellos, Krishna está en cada ser viviente, por lo tanto, mediante el servicio pretenden aportar al cambio personal, sociocultural y fomentar una relación recíproca entre los humanos y la naturaleza.

Figura 1. Dinámica del servicio según devotos Hare Krishna

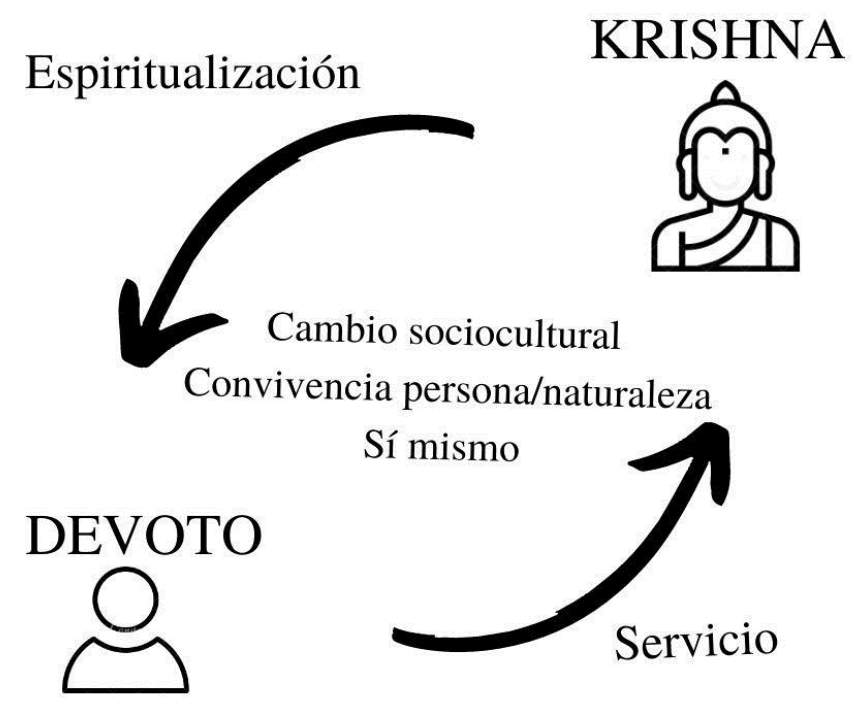

Fuente: elaboración propia basada en nuestro trabajo de campo y entrevistas a devotos Hare Krishna.

Mardones, R. y Díaz, P. (2021), Identidad, convivencia y espiritualidad en una comunidad ecológica Hare Krishna. Revista Cultura y Religión, 15(2), 244-272. 


\section{Directrices espirituales para un cambio sociocultural}

Las convicciones espirituales de nuestros participantes no solo se circunscriben a la vida comunitaria, sino que también están orientadas como una crítica y transformación positiva de nuestra sociedad.

Durante las entrevistas, nos narraron que en su trayectoria de vida tomaron conciencia sobre las consecuencias negativas de las directrices del mercado, situación que les impulsó a buscar nuevas alternativas de coexistencia alejadas del sistema neoliberal que, según nos contaron, tiene "hecha pedazos" la ecología, la sociedad y al ser, debido a la priorización de la economía, y el descuido del bien común de sus ciudadanos.

(...) no estaría tan inmerso en todo un sistema neoliberal que nos tienen hechos pedazos (...). La sociedad actual se basa solo en explotar sin cuidar lo esencial, entonces perturba la misma búsqueda de la felicidad en cada uno de nosotros, entonces, en realidad, como que la sociedad actual es una suciedad, porque no tienen conceptos muy limpios, ni propósitos muy armoniosos: es explotadora. Demasiado (...). (P.V, 2019, par. 1) ${ }^{1}$

Lo anterior nos remite a los cuestionamientos de las bondades y certezas inconclusas que prometía la modernidad (Thumala, 2003), a los que adhiere nuestro participante respecto de la sociedad contemporánea. Su diagnóstico crítico los lleva a caracterizar la sociedad como explotadora y restrictiva para el bienestar de las personas, aproximándose a lecturas contrarias al proyecto moderno y que advierten de la perversidad de sus consecuencias (Beriain, 1996; De la Cuadra, 2015).

Por otro lado, son cuestionadas las certezas que entrega el proyecto moderno, ya que, a ojos de un participante, en nuestra sociedad predomina una sensación de relatividad, que implica una clara limitación a la posibilidad de adquirir un conocimiento sólido y objetivo sobre el mundo. Esta falta de

\footnotetext{
${ }^{1}$ Las iniciales "P.V" o "M.V." identifican a nuestro/as participantes. El año "2019" señala la fecha en que fue enunciada la cita y "par." representa el número del párrafo en nuestro análisis.
}

Mardones, R. y Díaz, P. (2021), Identidad, convivencia y espiritualidad en una comunidad ecológica Hare Krishna. Revista Cultura y Religión, 15(2), 244-272. 


\section{Revista Cultura y Religión Vol. XV, Nº 2, (julio-diciembre) 2021}

certezas es contrarrestada con el conocimiento de Krishna y sus enseñanzas plasmadas en la Bhagavad Gita, su libro sagrado (García, 2018).

Los participantes mantienen la convicción de que en las enseñanzas de Krishna encuentran las orientaciones para la transformación social y nuevos modos de subsistencia. Esto implica un cambio personal basado en la ecología y la espiritualidad, en donde el desarrollo espiritual es el camino que orienta sus prácticas de articulación con los otros para la transformación social. Uno de los visitantes de la comunidad interpreta lo anterior como un elemento que cohesiona al grupo, y lo compara con "las aves del mismo plumaje [que] vuelan juntas". Esto también era interpretado por los/as voluntarios/as como personas con la misma vibración, sueños y cuestionamientos (nota de campo) ${ }^{2}$. Esta apropiación del discurso del movimiento Hare Krishna representa un instrumento para el cambio sociocultural, basado en la transformación personal (Vallverdú, 2001).

Nuestros participantes nos mencionan la desconexión espiritual que existe en la humanidad en esta época, ejemplificando que las prácticas habituales de las personas se basan en cosmovisiones antropocéntricas, no considerando perspectivas que promuevan la vida. Esta perspectiva biocéntrica asume la vida como centro y punto de partida de todas las disciplinas y comportamientos humanos (Puyol, 2001). Entonces, según nos relatan, existe escasa conciencia de totalidad, expresada en prácticas que obstaculizan la conexión del sí mismo y el cuidado de los ecosistemas de nuestro planeta.

Buscar la espiritualidad interna; tener una relación más amorosa entre todos los seres (...), seres humanos, seres animales, seres plantas, seres rocas, seres tierra, seres aire. Si encontramos esa relación más amorosa, nos va a empezar a importar que hagan un hoyo para sacar todos los minerales de la tierra en la punta del cerro, porque como las rocas dan lo mismo, porque no tienen vida (...) la mentalidad social del mundo occidental es así, fuerte. (M.V, 2019, par. 11)

\footnotetext{
${ }^{2}$ La expresión "nota de campo" refiere al registro de la observación participante realizada en el trabajo de campo.
}

Mardones, R. y Díaz, P. (2021), Identidad, convivencia y espiritualidad en una comunidad ecológica Hare Krishna. Revista Cultura y Religión, 15(2), 244-272. 


\section{Revista Cultura y Religión Vol. XV, Nº 2, (julio-diciembre) 2021}

Esta crítica ya no se dirige a una estructura social abstracta, sino que a la espiritualidad interna de cada sujeto que compone la sociedad. Este desplazamiento de la crítica considera un trabajo personal sincero, que alienta una reflexión sobre nuestra actuación personal en el mundo. Por ejemplo, un participante nos refiere lo siguiente: "un día vine, medité en lo que estoy haciendo y me di cuenta de que no iba pa' ningún lado" (P.V, 2019, par. 10). Se trata de identificar la falta de sentido de la rutina, el trabajo y la vida (nota de campo), y de las constricciones socioinstitucionales propias de nuestra sociedad (Beriain, 1996).

Finalmente, estas convicciones sobre la necesidad de un cambio sociocultural, mediante la práctica de una vida espiritual, se concreta en la cotidianidad comunitaria. Sus objetivos son prácticos, y su organización interna fomenta la articulación de las comunidades y fincas ecológicas de Chile y el mundo, para contribuir a una nueva sociedad basada en sus principios.

\section{Convivencia con las personas y la naturaleza}

La comunidad se fundó hace 18 años, aproximadamente. Sin embargo, nuestros participantes conviven en comunidad hace tres años. Decidieron optar por este estilo de vida, debido a que valoran las comunidades intencionales, ya que las perciben como lugares que propician la búsqueda del equilibrio, servicio y bienestar; aspectos que consideran importantes para enfrentar la explotación de la sociedad actual.

Los participantes nos relatan la importancia de convivir con personas que ayuden a sostener lo que ellos consideran una rebeldía, y que se animen a mantener la espiritualidad y el buen vivir (nota de campo). Uno de los devotos señala que “(...) la comunidad tiene ese propósito, de mejorar, de armonizar, de progresar, de proyectar, de simplificar la vida, y de servir al resto (...)" (P.V., 2019, par. 1). El propósito del que nos habla el devoto no es una intención particular de esta comunidad, sino que se articula con iniciativas rurales Hare Krishna en varias partes del mundo, en donde se estimula que cada devoto viva

Mardones, R. y Díaz, P. (2021), Identidad, convivencia y espiritualidad en una comunidad ecológica Hare Krishna. Revista Cultura y Religión, 15(2), 244-272. 
de acuerdo con los principios filosóficos védicos en armonía con la naturaleza y en compañía de personas que piensan similar acerca de Dios (Krishna), el propósito de vida y la transformación del mundo (Ávila, 2013).

En la convivencia comunitaria se busca llevar a la práctica el idealismo de una transformación social basada en la paz mundial, la sustentabilidad ecológica y el cuidado del cuerpo, alma y espíritu, promoviendo una sociedad bajo estos preceptos (Pulido- Muñoz, 2018: Sager, 2017). Observamos que se esfuerzan por concretar estos ideales a través de encuentros sociales abiertos, visitas guiadas a templos, celebraciones de festividades religiosas y almuerzos saludables abiertos a la comunidad los días domingo de cada semana. La literatura destaca la relevancia de la alimentación como un hecho social y cultural de carácter colectivo, que además se encontraría ligada a otras dimensiones de la vida social como la salud, la subsistencia y la organización social (Nuño, 2017).

Asimismo, los participantes mencionan la importancia de mostrar un ejemplo de estilo de vida ante la sociedad actual que contribuya a superar el miedo que la mayoría de las personas suelen sentir al optar por una vida alternativa (nota de campo). Interpretamos que lo anterior se relaciona con iniciativas locales que confrontan al sistema vigente desde adentro, alcanzando una escala microsocial (Silvestri, 2015). Para lograrlo, nuestra participante propone prácticas diversas que permiten tomar conciencia y que facilitan que otras personas se acerquen al movimiento.

(...) Estas generan conciencia de la tierra; conciencia de la madre, una conciencia de nuestro origen, una conciencia como desde adentro hacia afuera. Bueno, primero es desde afuera hacia adentro; mucha gente se acerca por el temazcal, por la huerta, por la bioconstrucción, y entremedio acá, se les da alimento que es ofrecido [a Krishna] primero, y después ven que cantamos mantras y, "oh mira son Hare Krishna" (risas). Entonces, de a poco como que la gente le va tomando el gusto a todo eso, a venir más seguido, a querer vivir de esta manera, a ver que es posible vivir de esta

Mardones, R. y Díaz, P. (2021), Identidad, convivencia y espiritualidad en una comunidad ecológica Hare Krishna. Revista Cultura y Religión, 15(2), 244-272. 


\section{Revista Cultura y Religión Vol. XV, Nº 2, (julio-diciembre) 2021}

manera, porque en el fondo muchas personas tienen miedo (...). (M.V, 2019, par. 3)

Observamos que estas prácticas se sostienen en una flexibilidad que no se aferra a ningún paradigma en concreto. En cambio, se expresa cierto sincretismo al fusionar el conocimiento veda y el del pueblo mapuche, por ejemplo. Según los participantes, la espiritualidad es única, difícilmente separable por culturas, por lo que recurren a conocimientos diversos, sin discriminar de qué cultura provengan.

(...) ¿Conocimientos a los que recurrimos? Conocimientos ancestrales, de la tierra, de pueblos indígenas, conocimientos de la cultura védica... como filosóficos digamos, y los dos se entremezclan como en la espiritualidad. Creo que va de la mano. Hay gente que lo puede separar, pero yo siento que van muy juntos, para mí no es separable (...). (M.V., 2019, par. 6)

Esta espiritualidad abierta, híbrida y flexible, se ha relacionado con el intercambio cultural entre maestros espirituales nativos y buscadores espirituales posmodernos, realizando diferentes prácticas extraídas de su contexto sociocultural (De la Torre, 2014). Esto facilita el surgimiento de una religión personalizada que se acopla a los estilos de vida de cada colectividad o participante (Farkas, 2018). En nuestro caso de estudio vemos que esta espiritualización de la religión facilita que los conocimientos diversos estén disponibles para la definición de su propia identidad.

Por un lado, la construcción de identidad está orientada por rituales y escrituras sagradas como la Bhagavad-Gita y otros libros de difusión Hare Krishna. Por otro lado, la actitud de servicio a la humanidad es un principio fundamental para la vida comunitaria de nuestros participantes. Cada vez que llega una visita o voluntario a la finca, mantienen esta actitud, reforzando su identidad a través de una narración sobre sí mismos y sus convicciones. La llegada de una visita a la comunidad es muy especial para ellos, ya que les permite poner en escena el servicio mediante preparaciones culinarias variadas,

Mardones, R. y Díaz, P. (2021), Identidad, convivencia y espiritualidad en una comunidad ecológica Hare Krishna. Revista Cultura y Religión, 15(2), 244-272. 
cantos, bailes y actividades que involucran al visitante en las dinámicas de la comunidad (nota de campo).

(...) mira, los que estamos ahora somos súper sociables, somos súper comunicativos, somos súper serviciales, pero si hubiese más gente (...) a veces hay gente que no le cuadra mucho, que no le agrada. Entonces sería como eso, nosotros ahora, estamos preocupados de toda la gente, somos muy serviciales (...). (P.V, 2019, par. 29)

Esta actitud servicial tiene como fin último servir a Krishna, y le entrega sentido a las prácticas cotidianas. “(...) Entonces, esa es como la meta que uno tiene: poder ser amable, ser amoroso, poder servir, ser comunitario. Entonces eso es como lo que a mí me inspira y me motiva (...)" (P.V, 2019, par. 64).

Nos llama la atención que el sentido de comunidad se encuentra en las prácticas de servicio que destacan nuestros participantes. Esto involucra el protagonismo del otro en la definición de su identidad, cuestión que puede involucrar la identificación y comparación con los miembros del grupo (Iñiguez-Rueda, 2001; Tajfel y Turner, 2004). De esta forma, el sujeto se considera a sí mismo como un ser en relación, que posee diferentes modos de ser y pensar la propia identidad (Gómez, 2003).

\section{Espiritualidad Hare Krishna en la definición de sí mismo}

Durante nuestro trabajo de campo observamos una construcción difusa de lo que ellos narran sobre su identidad actual, debido a que durante su trayectoria de vida fueron miembros de diferentes grupos sociales de pertenencia, en donde anclaban su identidad cada cierto tiempo, como, por ejemplo, en grupos anarquistas, evangélicos, punks, hippies, artistas callejeros, artesanos, terapeutas holísticos, entre otros (notas de campo). Sin embargo, estos diferentes anclajes identitarios muestran remanentes, los cuales aún se expresan en diferentes comentarios y comportamientos.

Mardones, R. y Díaz, P. (2021), Identidad, convivencia y espiritualidad en una comunidad ecológica Hare Krishna. Revista Cultura y Religión, 15(2), 244-272. 


\section{Revista Cultura y Religión Vol. XV, Nº 2, (julio-diciembre) 2021}

Coexiste la socialización en nuestro mundo y la intención de transformarse sobre la base de preceptos espirituales. Observamos roles tradicionales en la división social del trabajo, por ejemplo, nuestro participante aporta con el sustento económico a la familia a través de ventas ambulantes en la ciudad, y nuestra participante se dedica al trabajo doméstico, la crianza de sus hijos y la organización de los voluntariados.

La trayectoria personal de cada devoto nos permite observar que existe un esfuerzo por cambiar distintas dimensiones de su vida anterior al iniciarse como devotos de Krishna. La percepción de sí mismos era distinta y su conversión les permitió marcar un antes y después en su identidad. Por ejemplo, uno de nuestros participantes afirmó: “(...) era punk, era súper drogo, viajaba por todos lados, vivía en casas abandonadas, era ultraviolento (...)” (P.V, 2019, par. 48). Mientras que nuestra participante señala que su adhesión a los principios Hare Krishna le permitió un control sobre sí misma: “(...) yo antes de conocer [la] conciencia de Krishna, era la persona más explosiva que existía (...)" (M.V, 2019, par. 28).

No obstante, a medida que su aproximación a la espiritualidad avanza, la percepción de su identidad cambia. La percepción que ellos tienen de sí mismos se sitúa en una contingencia, que es vivir actualmente en esta comunidad. En consecuencia, la narración de su identidad se va construyendo en relación con una espiritualidad con potencialidad de cambio de sí mismo. Por ejemplo, se expresa en convicciones como: “(...) ya no soy tan loco, (...) estoy más tranquilo, (...) soy más responsable, (...) ven que yo estoy mejor, me ven feliz. Antes me veían enojado (...)” (P.V, 2019, par. 51).

La definición de la identidad desafía la noción del sujeto moderno al ampliar la idea de conciencia a otras esferas de realidad. Por ejemplo, mediante la atención al cuerpo o a sus relaciones con los otros: “(...) trato como de masticar, pensar, respirar y ver de qué manera puedo explicar lo otro de forma que no le afecte. Que no hiera el ego, porque cuando uno pasa a raspar el ego del otro, como que se engrifa (...)" (M.V, 2019, par. 28).

Mardones, R. y Díaz, P. (2021), Identidad, convivencia y espiritualidad en una comunidad ecológica Hare Krishna. Revista Cultura y Religión, 15(2), 244-272. 


\section{Revista Cultura y Religión Vol. XV, Nº 2, (julio-diciembre) 2021}

Otros elementos relevantes en la construcción de identidad de nuestros participantes se grafican en prácticas diarias que buscan construir lo que ellos quieren para sí mismos. En particular, sostienen una alimentación vegana, vestimentas ad hoc y el servicio devocional a Krishna.

Dicha alimentación vegana, basada en plantas y semillas, busca resguardar la biodiversidad y evitar el sufrimiento (nota de campo). Esto se justifica también por la convicción de que Krishna está en todas partes, y también está dentro de cada persona, animal y cosa (Cantú, 2019). De esta forma, el alimento para ellos es un acto de humildad y servicio; le cocinan a un ser supremo (Krishna) con el objetivo de que este bendiga los alimentos. Mientras más gente haya en la mesa, mejor; la hora de la comida se convierte en un acto de reunión, ya que se diferencia del acto de comer para la autosatisfacción; por el contrario, es un acto de servicio y devoción, al estar ingiriendo alimentos bendecidos y sin sufrimiento. Critican la vida acelerada y la "hipersatisfacción" de los sentidos, propios de la vida de la ciudad (nota de campo), lo cual inscriben en el sufrimiento, infelicidad y desorden del mundo material (Alcántara, 2019).

Lo anterior tiene como reverso el acto de cocinar. La literatura ha reportado que, para los devotos, el acto de cocinar es muy importante, incluso más que comer, porque este acto ritual implica un proceso de entender que la comida es para alguien más, para Dios; se renuncia al placer individual, para trascender el egoísmo y favorecer una vida en comunidad (Alcántara, 2019).

Otra particularidad en la práctica del comer es que no comparten sus alimentos, ni sus utensilios una vez servida la comida, ya que señalan que a través de la saliva se transmite el karma (nota de campo); cuestión que ha sido asociada a quebrantar las ideas de orden y armonía universal. La práctica del comer contribuiría a la no violencia (no comer carne); el control de los sentidos gustativos (no sentir placer egoísta); y, por último, el servicio a Dios y a los devotos, es decir, cocinar debe hacerse en estado de consciencia en Dios y en los miembros de la comunidad (Alcántara, 2019).

Mardones, R. y Díaz, P. (2021), Identidad, convivencia y espiritualidad en una comunidad ecológica Hare Krishna. Revista Cultura y Religión, 15(2), 244-272. 
Por otro lado, en relación con la vestimenta e indumentarias, observamos una distinción de género entre los/as participantes. Por ejemplo, no observamos a ninguna devota con pantalón dentro de la comunidad, sino que usaban faldas largas. Argumentan que los pantalones apretados interfieren en la conexión del útero con la madre tierra, unión importante para ellas. Además, en ceremonias usan los "saris" (palabra que significa "el envoltorio de una joya), vestidos generalmente traídos de la India,", y los "bindis" (punto en la frente) para diferenciar a las mujeres casadas de las solteras (nota de campo). Los hombres se cortan el pelo al ras, pero conservan un mechón en la parte posterior de la cabeza, la "shika". Este mechón o shika lo mantienen para conectarse con la dimensión espiritual, ya que por el chakra de la corona -un punto físico que se ubica sobre la cabeza- es por donde entra la energía. Ambos sexos se pintan una "V" en la frente en festividades o ceremonias, lo que significa adoración a Vishnú; Krishna es la encarnación de Vishnú. (notas de campo).

Por último, la devoción hacia Krishna incita a nuestros participantes a entregar su servicio a la biodiversidad y la humanidad. Cada cierto tiempo realizan encuentros sociales. Por ejemplo, una de sus actividades comunitarias más importantes es el Chaski Fest, el cual reúne a personas de las ciudades y poblados cercanos a la comunidad para tratar problemas ambientales y espirituales de forma abierta por medio de diversas expresiones artísticas.

Durante el año intentan transmitir las enseñanzas de Krishna en actos, los que se traducen en talleres sobre autocuidado y desarrollo espiritual, encuentros de reflexión sobre el sentido de vida y el cuidado de la naturaleza, la transmisión de saberes ancestrales de la tierra, y la promoción del vegetarianismo, mediante talleres de alimentación consciente, distribución de recetas vegetarianas y almuerzos comunitarios abiertos (notas de campo). Además, cultivan una actitud amorosa, servicial y de apertura comunitaria, junto al cuidado de la familia como espacio de servicio a Krishna.

El ascetismo que siguen los devotos, expresado en la adopción de vestimentas e indumentarias de identificación, cortes de cabellos, estrictas dietas vegetarianas, alimentación como ritual y la renuncia o restricción de sus

Mardones, R. y Díaz, P. (2021), Identidad, convivencia y espiritualidad en una comunidad ecológica Hare Krishna. Revista Cultura y Religión, 15(2), 244-272. 


\section{Revista Cultura y Religión Vol. XV, Nº 2, (julio-diciembre) 2021}

vínculos sociales de origen (familia, escuela, amigos de barrio, etc.), así como a todo aquello que tenga relación con la explotación propia de la cultura occidental, influyen de manera significativa en la reconstrucción de su identidad (Bahamondes y Marín, 2015; Vallverdú, 1999). Sin embargo, según nuestro trabajo en terreno, observamos que en nuestra comunidad no abandonan radicalmente los vínculos sociales de origen, ya que continúan manteniendo viejas amistades, las que son invitadas a la comunidad para ocasiones especiales como, por ejemplo, la celebración de sus cumpleaños (notas de campo).

\section{Conclusiones}

La crisis sistémica trae consigo cuestionamientos e incertidumbres que no solo se dirigen a uno mismo, sino que también ponen en duda el futuro del planeta, ante la evidencia innegable de la degradación ambiental y problemáticas sociales como la pobreza, hambrunas y la violencia; o la degradación personal expresada en el individualismo y hedonismo de nuestra sociedad. En este contexto, nuestros participantes ejemplifican la búsqueda y concreción de un estilo de vida alternativo como respuesta a la crisis sistémica (Bahamondes y Marín, 2015; Bauman, 2001), lo cual impacta en la constitución de su identidad.

Nuestros participantes explican la constitución de su identidad como un proceso dinámico que gira en torno a la figura de su deidad: Krishna. Gracias a una conversión espiritualizada de sus creencias, vuelcan su vida al servicio para conseguir una transformación de sí mismos, de sus relaciones con otras personas y con la naturaleza, así como también, un cambio en nuestra sociedad.

En particular, vemos cómo las convicciones religiosas orientan sus directrices espirituales hacia un cambio sociocultural, buscando su lugar para contribuir de forma práctica a través del ejemplo y el servicio devocional. Esto tiene un correlato práctico, ya que consideran que sirven a un dios que está en el

Mardones, R. y Díaz, P. (2021), Identidad, convivencia y espiritualidad en una comunidad ecológica Hare Krishna. Revista Cultura y Religión, 15(2), 244-272. 
interior de cada ser, planta y animal, lo que promueve la protección de formas de vida humanas y no-humanas (King, 2012).

En lo cotidiano, distinguimos que existe una apropiación y traducción de las orientaciones religiosas, expuestas en su libro sagrado, para modular la convivencia con los otros. Los testimonios de nuestros participantes no son homogéneos, y sus explicaciones están integradas en un relato sobre su propia trayectoria biográfica. Es en este punto en donde constatamos que la espiritualidad Hare Krishna, sobre la que se constituyen como sujetos, mayoritariamente se articula en la acción respecto a otro. El otro no es solo una persona, sino que lo puede ser cualquier ser vivo, en donde potencialmente se reencarne el alma de un ser, lo que conlleva a una sensibilidad y respeto especial por todo lo que les rodea.

En lo cotidiano, el servicio es central. Se expresa, por ejemplo, en un liderazgo distribuido, una alimentación vegana, cocinar para otros o convocar actividades abiertas para promover el cuidado del medioambiente. Estas prácticas entregan dinámicas diarias en las que nuestros participantes construyen su identidad, en función de los modos de ser y pensar que están configurados por la interacción que mantienen con los devotos semejantes de la comunidad. Es decir, su identidad también incluye creencias trasmitidas por sus semejantes y actúan sobre la base de esta percepción con el propósito de cumplir con las expectativas de los otros y de ellos mismos (Merton y Merton, 1968). En nuestros hallazgos constatamos que la narración de los devotos acerca de prácticas ascéticas, como el no tener relaciones sexuales por placer, el veganismo, meditaciones, etc., muestran flexibilidad, en contraste a lo que la literatura señala sobre un estricto código moral de comportamiento para trascender el materialismo y la mortificación del ego (Vallverdú, 2001)

Es central para los devotos la conexión consigo mismos y la claridad de quiénes son y cuáles son sus propósitos, ya que reportan que esto es importante para aportar a los cimientos de una nueva sociedad, es decir, sus convicciones nos indican que mientras más personas tengan un cambio personal, mayor será la posibilidad de un cambio social. De esta forma, la construcción de identidad

Mardones, R. y Díaz, P. (2021), Identidad, convivencia y espiritualidad en una comunidad ecológica Hare Krishna. Revista Cultura y Religión, 15(2), 244-272. 
basada en principios Hare Krishna se interpreta como un modo de transformación social, que se sustenta en la idea de una conexión espiritual individual y comunitaria, expresada en prácticas cotidianas. Por ejemplo, la mortificación del ego mediante el servicio devocional a Krishna, la valorización de la vida a través del veganismo, y el cuidado del ecosistema y la autorrealización espiritual.

La espiritualidad Hare Krishna es la articuladora de la definición de sí mismo (Squarcini, 2000). Para nuestros participantes, Krishna está en el interior del sujeto, lo que implica que también deben servir a su cuerpo, alma y espíritu. Por consecuencia, se da una interdependencia que muestra que al mismo tiempo que la persona trabaja en el desarrollo de su conciencia, cambia sus relaciones cotidianas, cambia la sociedad y sirve a Krishna.

Concluimos que la actitud de servicio es la característica más destacada para responder a nuestra pregunta sobre la identidad. Reconocemos en el cultivo de prácticas espirituales la potencia de repensar otras opciones de vida, alejadas del individualismo y consumismo, elementos propios de la modernidad. Consideramos que nuestro caso de estudio revitaliza la discusión sobre las posibilidades de relaciones simétricas (Latour, 2008), ya que los límites comienzan a diluirse en una comprensión unitaria de la persona, la sociedad y la naturaleza. Finalmente, constatamos flexibilidad en las constricciones de una vida cotidiana sostenida en convicciones dogmáticas. Sin embargo, no podemos ignorar algunas contradicciones que muestran la reproducción de prácticas tácitas en torno a las relaciones socioeconómicas y de género, las cuales les ofrecen un reto para fortalecer su perspectiva del cambio sociocultural.

\section{Referencias bibliográficas}

Alcántara, E. (2019). La cocina vaishnava: aproximación etnográfica a la alimentación espiritual Hare Krisna. (Tesis para optar a la maestría en Antropología Social y Política). FLACSO, Buenos Aires, Argentina.

Mardones, R. y Díaz, P. (2021), Identidad, convivencia y espiritualidad en una comunidad ecológica Hare Krishna. Revista Cultura y Religión, 15(2), 244-272. 
Alsina, R. y Bravo, P. (2006). Posmodernidad y crisis de identidad. IC Revista científica de información y comunicación, (3), 125-146.

Álvarez-Munárriz, L. (2011). La compleja identidad personal. Disparidades. Revista de Antropología, 66(2), 407-432. DOI: 10.3989/rdtp.2011.15

Ávila, C. (2013). Crónicas vaisnavas: una mirada de la filosofía Hare Krishna. (Tesis para optar al título de periodista). Universidad de Chile, Santiago, Chile.

Bahamondes, L. y Marín, N. (2015). Expansión Hare Krishna en contextos de crisis: resignificando la movilización social desde una perspectiva religiosa en Chile. Disparidades. Revista de Antropología, 70(2), 379398. DOI: 10.3989/rdtp.2015.02.004

Bauman, Z. (2001). La posmodernidad y sus descontentos. Madrid: Ediciones Akal.

Bauman, Z. y Tester, K. (2002). La ambivalencia de la modernidad y otras conversaciones. Barcelona: Ediciones Paidós.

Beck, U., Giddens, A. y Lash, S. (1997). Modernidad reflexiva. Barcelona: Península.

Berger, P. y Luckmann, T. (1997). Modernidad, pluralismo y crisis de sentido: la orientación del hombre moderno. Barcelona: Ediciones Paidós.

Beriain, J. (comp). (1996). Las consecuencias de la perversa modernidad. Barcelona: Anthropos.

Bögre, Z. (2016). Conversion, personal identity, life history. Sociologie Românească, 14(04), 79-88.

Cantú, J. (2019). Sociología clínica: ¿Qué es el sufrimiento social? Anuario Humanitas, 0(44), 113-128.

Carnevali, S. (2009). La representación social de los Hare Krishna: miedo, intolerancia, y discriminación hacia las minorías religiosas de tipo sectarias. (Tesis para optar al grado de Licenciatura en Historia). Universidad de Chile, Santiago, Chile.

Castells, M. (1999). Globalización, identidad y estado en América Latina. Recuperado comunidad ecológica Hare Krishna. Revista Cultura y Religión, 15(2), 244-272. 
https://flacsoandes.edu.ec/sites/default/files/agora/files/1266426228.glo balizacion_castells.pdf [Consultado el 12 de julio de 2019].

Dabat, A. (2002). Globalización, capitalismo actual y nueva configuración espacial del mundo. En J. Basave, A. Dabat, C. Morena, A. Rivera y F. Rodríguez (coords), Globalización y alternativas excluyentes para el siglo XXI (pp. 41-88). México: Universidad Autónoma del Estado de Morelos.

De la Cuadra, F. (2015). Buen vivir: ¿una auténtica alternativa post-capitalista? Polis, Revista Latinoamericana, 14(40), 7-19. DOI: 10.4067/S071865682015000100001

De la Torre, R. (2014). Los Newagers: el efecto colibrí. Artífices de menús especializados, tejedores de circuitos en la red, y polinizadores de culturas híbridas. Religião e Sociedade, 34(2), 36-64. DOI: 10.1590/S1984-04382014000200003

Erlandson, D., Harris, E., Skipper, B. y Allen, S. (1993). Doing Naturalistic Inquiry: A Guide to Methods. Londres: SAGE Publications.

Estermann, J. (2012). Crisis civilizatoria y vivir bien. Una crítica filosófica del modelo capitalista desde el allin kawsay/suma qamaña andino. Polis, Revista Latinoamericana, 11(33), 149-174. DOI: 10.4067/S071865682012000300007

Farkas, J. (2018). Nature faith and native faith as integrative spiritualities in Hungarian ecovillages. Journal for the Study of Religion, Nature and Culture, 12(2), 125-146. DOI: 10.1558/jsrnc. 29630

Ferreira, C. (2013). Modernidade: rupturas com a tradicao. Diálogos. Revista de estudos culturais e da contemporanidade, 8(1), 119-134.

García, A. (2018). Hare krishna: una mirada hacia el interior del ser. (Tesis para optar al título de Comunicadora Social). Pontificia Universidad Javeriana, Bogotá, Colombia.

Gergen, K. (2006). El yo saturado: dilemas de identidad en el mundo contemporáneo. Barcelona: Paidós.

Mardones, R. y Díaz, P. (2021), Identidad, convivencia y espiritualidad en una comunidad ecológica Hare Krishna. Revista Cultura y Religión, 15(2), 244-272. 


\section{Revista Cultura y Religión Vol. XV, № 2, (julio-diciembre) 2021}

Gómez, S. (2003). La ocupación y su significado como factor influyente de la identidad personal. Revista Chilena de Terapia Ocupacional, (3), 43-47. DOI: $10.5354 / 0719-5346.2010 .149$

Granados, J. (2019). Méndez, Á. Festín del deseo. Hacia una teología alimentaria. Cultura \& religión, 13(1), 125-128. https://dx.doi.org/10.4067/S0718-47272019000100125

Granados, J. (2020). Estética de la alegría del sacrificio. Anacronismo de la modernidad. Cultura \& Religión, 14(1), 13-25. DOI: 10.4067/S071847272020000100013

Hall, S. (2011). Introducción: ¿Quién necesita una identidad? En S. Hall y P. du Gay (comps.), Cuestiones de Identidad cultural (pp. 13-39). Buenos Aires: Amorrortu.

Herrera, E. (2018). Análisis de la relación entre el concepto humanista de la búsqueda de sentido y las personas que deciden convertirse en devotos de la Sociedad Internacional para la Conciencia Krishna. (Tesis para optar el título de Psicología Clínica). Pontificia Universidad Católica del Ecuador, Quito. Ecuador.

Huiliñir-Curío, V. y Zunino, H. (2017). Movilidad, utopías y lugares híbridos en Los Andes del sur de Chile. Revista INVI, 32(91), 141-160. DOI: 10.4067/S0718-83582017000300141

Ibáñez, T. (2001). Municiones para disidentes: realidad, verdad, política. Barcelona: Gedisa Editorial.

Iñiguez-Rueda, L. (2001). Identidad: de lo personal a lo social. Un recorrido conceptual. En E. Crespo y C. Soldevilla (eds.), La constitución social de la subjetividad (pp. 209-225). Madrid: Catarata.

Kawulich, B. (2005). La observación participante como método de recolección de datos. FQS, 6(2), 1-32.

King, A. (2012). Krishna's Cows: ISKCON's Animal Theology and Practice. Journal of Animal Ethics, 2(2), 179-204. DOI: 10.5406/janimalethics.2.2.0179

Mardones, R. y Díaz, P. (2021), Identidad, convivencia y espiritualidad en una comunidad ecológica Hare Krishna. Revista Cultura y Religión, 15(2), 244-272. 


\section{Revista Cultura y Religión Vol. XV, Nº 2, (julio-diciembre) 2021}

Kozeny, G. (1995). Intentional communities: Lifestyles based on ideals. Recuperado de: http://articles-andessays.s3.amazonaws.com/Intentional+community+/G-Kozenylifestyles-based-on-ideals.pdf [Consultado el 10 de julio de 2019].

Latour, B. (2008). Reensamblar lo social. Una introducción a la teoría del actor-red. Buenos Aires: Manantial.

Lerner, V. (2019). Los sentidos de pertenencia de los y las jóvenes que participan en el Movimiento Masortí en la ciudad autónoma de Buenos Aires. Percepciones sobre el movimiento y la "comunidad". Cultura \& Religión, 13(1), 64-84. DOI: 10.4067/S0718-47272019000100064

Luarte, F. (2017). Breve historia de la religiosidad hindú en Chile. Revista del CESLA. International Latin American Studies Review, (20), 245-260.

Mardones, R. y Zunino, H. (2019). Repensando lo comunitario: discursos de comunidades intencionales utópicas en Chile. Convergencia, Revista de Ciencias Sociales, 26(81), 1-21. DOI: 10.29101/crcs.v0i81.10615

Mardones, R. y Zunino, H. (2021). Emplazando la utopía: reinvenciones del sujeto, la comunidad y el espacio habitado en Chile. Revista de Geografía del Norte Grande (78), 49-69. DOI: 10.4067/S071834022021000100049

Mardones, R., Aguirre, R., Aranda, E. y Muñoz, J. (2019). Lugares transitorios para la reinvención del sujeto moderno. En C. Rojas, Drogas: Sujeto, sociedad y cultura (pp. 75-90). Talca: Nueva Mirada.

Mardones, R., Ulloa, J. y Salas, G. (2018). Usos del diseño metodológico cualitativo en artículos de acceso abierto de alto impacto en ciencias sociales. Forum Qualitative Sozialforschung/Forum: Qualitative Social Research, 19(1). DOI: 10.17169/fqs-19.1.2656

Merton, R. C. y Merton, R. K. (1968). Social Theory and Social Structure. Nueva York: Free Press.

Muñoz, E. (2018). Ecoaldeas en Colombia transitando hacia el buen vivir. Entramado, 14(2), 114-131. DOI: $\quad 10.18041 / 1900-$ 3803/entramado.2.4754

Mardones, R. y Díaz, P. (2021), Identidad, convivencia y espiritualidad en una comunidad ecológica Hare Krishna. Revista Cultura y Religión, 15(2), 244-272. 


\section{Revista Cultura y Religión Vol. XV, Nº 2, (julio-diciembre) 2021}

Nuño, N. (2017). Crisis alimentarias y religión en la modernidad tardía. Cambios, reinterpretaciones y mercantilización de los principios alimentarios en el culto Hare Krishna. Anales de Antropología, 51(1), 56-63. DOI: 10.1016/j.antro.2016.10.005

Pérez-Luco, R., Lagos, L., Mardones, R. y Sáez Ardura, F. (2017). Taxonomía de diseños y muestreo en investigación cualitativa. Un intento de síntesis entre las aproximaciones teórica y emergente. Ámbitos. Revista Internacional de Comunicación, (39), 1-18.

Pulido-Muñoz, L. (2018). Contribución a la paz en Colombia desde las prácticas y sentidos de paz desarrollados por la Ecoaldea Varsana en asociación con la red del pacto mundial consciente. Revista Aletheia, 10(1), 214-237. DOI: 10.11600/21450366.10.2aletheia.214.137

Puyol, Á. (2001). El discurso de la igualdad. Barcelona: Crítica.

Quintana, P. (2011). Chilenos convertidos en devotos de Krishna: una aproximación al movimiento internacional para la conciencia de Krishna en Santiago de Chile. (Tesis para optar al título de Antropología). Universidad Bolivariana, Santiago, Chile.

Rose, N. (2019). La invención del sí mismo: Poder, ética y subjetivación. Santiago: Editorial Pólvora.

Sager, T. (2017). Planning by intentional communities: An understudied form of activist planning. Planning Theory, 17(4), 449-471.

Salamanca, L. y Silva, D. (2015). El movimiento de ecoaldeas como experiencia alternativa de Buen Vivir. Polis, Revista Latinoamericana, (40), 1-19. DOI: 10.4067/S0718-65682015000100011

Sánchez, O., Plascencia, F. y Bernal, L. (2020). Grupo de referencia etario, régimen simbólico y religiosidad. La religiosidad como sistema abierto de creencias en estudiantes universitarios. Cultura \& Religión, 14(2), 121-140. https://dx.doi.org/10.4067/S0718-47272020000200108

Schuttenberg, M. (2007). Identidad y globalización. Elementos para repensar el concepto y su utilización en ciencias sociales. Cuaderno de H Ideas, 1(1), 1-20.

Mardones, R. y Díaz, P. (2021), Identidad, convivencia y espiritualidad en una comunidad ecológica Hare Krishna. Revista Cultura y Religión, 15(2), 244-272. 


\section{Revista Cultura y Religión Vol. XV, Nº 2, (julio-diciembre) 2021}

Silvestri, M. (2015). El propósito de vida en la Comunidad Intencional. La transformación personal para el cambio micro-social. (Tesis para optar al grado de doctor en Psicología Social). Universidad Autónoma de Barcelona, Barcelona, España.

Spink, M. (org.) (2013). Prácticas discursivas e produção de sentidos no cotidiano: Aproximações teóricas e metodológicas. São Paulo: Centro Edelstein de Pesquisas Sociais.

Squarcini, F. (2000). In search of identity within the Hare Krishna movement: memory, oblivion and thought style. Social Compass, 47(2), 253-271. DOI: $10.1177 / 003776800047002008$

Sullivan, E. (2016). Individualizing utopia: Individualist pursuits in a collective cohousing community. Journal of Contemporary Ethnography, 45(5), 602-627. DOI: 10.1177/0891241615587527

Tajfel, H. y Turner, J. (2004). The social identity of intergroup behavior. En J. Jost y J. Sidanius (eds.), Key Readings in Social Ppsychology. Political Psychology: Key Readings (pp. 276-293). Nueva York: Psychology Press.

Taylor, C. (2006). Fuentes del yo: La construcción de la identidad moderna. Barcelona: Paidós.

Thumala, D. (2003). Modernidad y sufrimiento: algunos elementos para la comprensión de la significación cultural de la experiencia del sufrimiento. Revista Mad, (8), 1-47. DOI: 10.5354/07180527.2011 .14801

Ulloa, J. y Mardones, R. (2017). Tendencias paradigmáticas y técnicas conversacionales en investigación cualitativa en ciencias sociales. Perspectivas de la Comunicación, 10(1), 213-235.

Vallverdú, J. (1999). Conversión, compromiso y construcción de identidad en el movimiento Hare Krishna. Alteridades, 9(18), 57-70.

Vallverdú, J. (2001). Movimientos religiosos e identidades juveniles. Hare Krishna en Occidente. Revista de Estudios de Juventud, 53(1), 57-71.

Mardones, R. y Díaz, P. (2021), Identidad, convivencia y espiritualidad en una comunidad ecológica Hare Krishna. Revista Cultura y Religión, 15(2), 244-272. 
Revista Cultura y Religión Vol. XV, Nº 2, (julio-diciembre) 2021

Zapata, H. (2019). Creer, experimentar, comprender: una reflexión sobre la intersubjetividad a propósito de una experiencia de intervención en el campo de la creencia. Cultura \& Religión, 13(1), 45-63. DOI: $10.4067 /$ S0718-47272019000100045

Mardones, R. y Díaz, P. (2021), Identidad, convivencia y espiritualidad en una comunidad ecológica Hare Krishna. Revista Cultura y Religión, 15(2), 244-272. 